\title{
Ações de saúde desenvolvidas pelo núcleo de apoio à saúde da família - NASF
}

\author{
Marcielly dos Santos Maciel'1, Márcia Oliveira Coelho², Lidia Audrey Rocha Valadas Marques³, \\ Edilson Martins Rodrigues Neto ${ }^{4}$, Mara Assef Leitão Lotif ${ }^{5}$, Emerson Dias Ponte ${ }^{6}$
}

\begin{abstract}
RESUMO
O Núcleo de Apoio à Saúde da Família (NASF) é uma estratégia que diversifica as ações das equipes de Estratégia de Saúde da Família (ESF). O presente estudo objetiva relatar as ações realizadas pelo grupo Revivendo do NASF junto à comunidade assistida pelo programa e pela ESF. Trata-se de relato de experiência de ações de saúde realizadas por um grupo - Grupo Revivendo - do NASF de uma Unidade de Atenção Primária à Saúde de Fortaleza - CE. As atividades ocorreram uma vez por semana, durante sete meses, totalizando 28 encontros, sendo conduzidas pelas equipes do Nasf e da ESF no período de março a novembro de 2011. A reunião de grupo tinha uma duração aproximada de 90 minutos, cujos participantes tinham a idade média de 60 anos. As atividades do "Grupo Revivendo" foram realizadas com pessoas de todas as faixas etárias, onde a educação em saúde foi a prioridade. Percebeu-se a importância da atuação dos profissionais do NASF e da atenção primária à saúde ao atravessa os limites das Unidades de Saúde, adentrando no território da comunidade, valorizando e fortalecendo as parcerias sociais.
\end{abstract}

Descritores: Promoção da Saúde; Atenção Primária à Saúde; Saúde da Família.

\section{Health actions taken by the support health center family - NASF}

\begin{abstract}
The Support Center for Family Health (NASF) is a strategy that diversifies the actions of the Family Health Teams (ESF). This study aims to report the actions taken by the group Reviving of NASF with the community assisted by the program and the ESF. This is an experience report of health actions performed by a NASF group - Reviving Group - of a Unit of Primary Health Care of Fortaleza - CE. The activities occurred once a week, for seven months, totaling 28 meetings, being conducted by Teams of NASF and the ESF in the period march to november 2011. The group meeting had an approximate duration of 90 minutes, which participants had an average age of 60 years. The activities of "Reviving Group" were conducted with people of all ages, in which health education was a priority. Was realized the importance of the NASF professionals performances and primary health care when crossing the Health Units frontiers, entering the community territory, valuing and strengthening the social partnerships.
\end{abstract}

Descriptors: Health Promotion; Primary Health Care; Family Healthy.

\footnotetext{
${ }^{1}$ Graduada em Fisioterapia pela Faculdade Integrada do Ceará (FIC), Brasil, Fortaleza, CE, Brasil.

${ }^{2}$ Mestre em Saúde Pública pela Universidade Federal do Ceará (UFC), Fortaleza, CE, Brasil.

${ }^{3}$ Especialista em Farmacologia Clínica pelo Instituto Ateneu, Fortaleza, CE, Brasil.

${ }^{4}$ Mestre em Farmacologia, Universidade Federal do Ceará (UFC), Fortaleza, CE, Brasil.

${ }^{5}$ Cirurgiã-dentista pela Universidade Federal do Ceará (UFC), Fortaleza, CE, Brasil.

${ }^{6}$ Graduado em Odontologia pela Universidade Federal do Ceará (UFC), Fortaleza, CE, Brasil.
} 


\section{Introdução}

O Programa Saúde da Família (PSF) criado pelo Governo Federal se consolidou como estratégia prioritária para reorganização da atenção básica de saúde. A partir dele surgiu a Política Nacional de Atenção Básica1.

A discussão acerca da implantação do Programa de Saúde da Família (PSF) em Fortaleza data de 1997, mas apenas em 1999 foram constituídas as primeiras equipes. Após a realização do concurso público do PSF em 2006, Fortaleza passou a ser considerada a terceira cidade do Brasil com maior cobertura do PSF, entre os municípios com mais de 1,5 milhão de habitantes, atingindo $35 \%$ da população da capital. A cidade fica atrás apenas de Belo Horizonte, com $75 \%$ da cobertura do PSF, e Recife, com $56 \% 2$.

Atualmente o termo PSF mudou para Estratégia Saúde da Família (ESF). A Estratégia de Saúde da Família é uma reorganização e reorientação do modelo assistencial, mediante a disposição de equipes multiprofissionais em unidades de saúde distribuídas em territórios delimitados espacialmente ${ }^{3}$.

Nessa lógica de organização territorial, o espaço físico da ESF deixou de ser meramente o espaço político-operativo do sistema de saúde, passando a ser um campo no qual se verifica a interação entre uma população específica, de um espaço determinado e os serviços no âmbito local. Essa população normalmente apresenta problemas de saúde definidos e interage com os gestores das distintas unidades prestadoras de serviços de saúde 4 .

O Núcleo de Apoio à Saúde da Família (NASF) é uma potente estratégia para ampliar a abrangência e diversidade das ações da ESF bem como sua resolubilidade, uma vez que promove a criação de espaços para a produção de novos saberes e ampliação da clínica. O NASF não se constitui porta de entrada do sistema para os usuários, mas apoio às equipes de saúde da família, vinculando a um determinado número de equipes e executando suas atividades em territórios definidos ${ }^{5}$.

A ampliação da clínica para além dos consultórios e dos muros das unidades de saúde, através da formação de grupos na comunidade, favorecem as trocas de saberes como estratégias de prevenção de doenças e busca ativa de doenças, principalmente as crônico degenerativas ${ }^{5}$.

Como importante doença crônica degenetiva a hipertensão arterial sistêmica (HAS) tem grande importância epidemiológica no Brasil, grande parte dos estudos encontrados relacionam a hipertensão arterial e educação em saúde a fim de promover maior adesão ao tratamento ${ }^{6}$.

De acordo com o Ministério da Saúde estima-se que mais de 15 milhões de brasileiros têm hipertensão arterial, sendo que mais de um $1 / 3$ desconhecem a doença e menos de $1 / 3$ dos hipertensos com diagnóstico apresentam níveis adequados de pressão arterial com tratamento proposto ${ }^{7}$.

Devido a seu grande impacto na morbimortalidade da população brasileira, o Ministério da Saúde implementou, em 2001, o Plano de Reorganização da Atenção à Hipertensão Arterial e ao Diabetes mellitus, tendo a educação e a promoção à saúde como alicerces. Desse modo, optou-se por abranger este público, pois, além de ser uma das prioridades para 0 Ministério da Saúde, sabe-se que a prevenção é bastante eficaz para o controle da HAS .

$O$ presente trabalho objetiva relatar as ações realizadas pelo grupo Revivendo do NASF junto à comunidade assistida pelo programa e pela ESF, pontuando as principais facilidades/dificuldades no desempenho das competências do NASF junto à comunidade assistida pela ESF e NASF da Cidade de Fortaleza - CE. Sendo assim, o estudo poderá contribuir para com os profissionais de saúde no intuito de se pontuar e compartilhar as ações exitosas e em contrapartida relacionar as dificuldades encontradas para se favorecer e/ou aprimorar estratégias terapêuticas.

\section{Metodologia}

Trata-se de um relato de experiência das atividades desenvolvidas pela equipe do NASF em um grupo de usuários da comunidade do bairro Bom Jardim realizado no período de março a novembro de 2011 na Unidade de Atenção Primária à Saúde Guarany Mont'alverne em Fortaleza-CE. As atividades ocorreram uma vez por semana, durante sete meses, totalizando 28 encontros. A reunião de grupo tinha uma duração aproximada de 90 minutos, cujos participantes tinham a idade média 60 anos.

O referido grupo recebeu o nome de "Revivendo" e foi composto por pacientes com hipertensão arterial sistêmica (HAS) de todas as faixas etárias e ambos os sexos que desejavam controlar a HAS (tendo uma frequência média de 32 participantes), usuários que desejavam prevenir esta doença e indivíduos que já apresentavam sequelas da mesma, como era o caso dos pacientes que haviam sofrido acidente vascular cerebral (AVC). 
Quando a equipe do NASF foi introduzida em 2011 na unidade de saúde, a equipe 1 da ESF realizava atendimento na comunidade para controle da pressão arterial e diabetes. No entanto, os encontros eram mensais e tinham como âmago a educação em saúde, a mensuração da pressão arterial e a verificação dos níveis glicêmicos, não estando inseridas as práticas corporais.

Como estratégia de aprimorar o atendimento aos hipertensos e diabéticos, pactuou-se iniciar um grupo que pudesse ser contínuo e que incluísse também práticas corporais como alongamentos, exercícios aeróbios, exercícios terapêuticos e dinâmicas que envolvessem músicas e danças com objetivo de prevenir e controlar os índices de hipertensão e suas complicações, como o AVC.

Para a implantação do grupo, reuniu-se a equipe do NASF formada por uma fisioterapeuta, uma educadora física, uma assistente social, um farmacêutico, uma fonoaudióloga e uma psicóloga e Equipe 1 da ESF da unidade de saúde formada por um médico, uma enfermeira e agentes comunitários de saúde (ACS). Os ACS,foram os responsáveis pela divulgação do grupo na comunidade. Iniciou-se o grupo com os usuários desta equipe, e, logo depois de alguns encontros realizados, os ACS das equipes da ESF 2 e 3 solicitaram a inclusão dos membros de sua comunidade que tiveram interesse em participar do grupo, no qual foram aceitos e acolhidos por todos os participantes.

As atividades foram desenvolvidas na Sociedade Beneficente Sara Rozita, local escolhido em comum acordo entre a equipe de Saúde da Família e equipe do NASF. A estrutura cooperou para o desenvolvimento do trabalho, pois contava com quadra de esportes, salas para atendimento coletivo e individuais com mesas, cadeiras e macas, sonorização que possibilitava incluir a música durante a dançoterapia.

\section{Resultados e discussão}

As atividades iniciavam-se com a mensuração da PAS de cada participante, após a verificação registrou-se os resultados na ficha de acompanhamento, diário de atividades e procedimentos da equipe NASF, esta ficha é um dos instrumentos de registro que a Secretaria Regional SER-V exige mensalmente às equipes de NASF. Portanto, todos os dados deste grupo encontram-se registrados e armazenados na SER-V no Distrito de Saúde.

Em um segundo momento, o grupo foi conduzido com ações educativas de práticas corporais, exercícios e aconselhamentos sobre o tratamento da HAS. Foi através dessas atividades de educação em saúde que se proporcionou às pessoas o desenvolvimento e o senso de responsabilidade pela sua própria saúde. As equipes do NASF e da ESF tiveram como objetivo levar para a comunidade benefícios do conhecimento e da prática de gestos e atitudes que poderiam melhorar a qualidade de vida da população.

A educação em saúde é um mecanismo de troca de saberes e/ou experiências que ocorre entre a população em geral, incluindo os usuários, profissionais e gestores de saúde, em que cada indivíduo é valorizado como dono de um saber. Esta prática visa à prevenção de doenças, à promoção da saúde e à autonomia dos sujeitos envolvidos, tornando-os ativos e transformadores de sua própria vida ou até mesmo da sua sociedade9.

Na realização da atividade educativa foram utilizados métodos dinâmicos, que se julgou ser o mais adequado para este tipo de atividade. Durante o processo educativo se evitou a monotonia e a repetição através de dinâmicas de grupo, desenhos, jogos educativos, músicas e danças.

O grupo estava aberto à participação dos outros profissionais de saúde, assistente social, fonoaudiólogo, psicólogo, farmacêutico, médico, enfermeiros e auxiliares de enfermagem quando solicitados, pois estes realizavam outras atividades como atendimentos nas unidades e visitas domiciliares no horário do grupo, no entanto os usuários eram encaminhados, quando havia necessidade.

Dentro deste contexto foram realizadas atividades educativas que explanavam formas de prevenir a hipertensão e seus malefícios. A interação era estimulada por meio da rodas de conversa, que priorizava o diálogo sobre a importância do controle da pressão arterial e as seque las que a hipertensão poderia trazer, como o AVC.

A prática utilizada - rodas de conversa - é uma estratégia de educação em saúde que tem como objetivo proporcionar um ambiente no qual os sujeitos podem trocar informações, experiências e vivências ${ }^{10}$.

Procurou-se mostrar os benefícios de uma alimentação saudável, o ensino de determinados exercícios terapêuticos. Associados a uma alimentação saudável, os exercícios terapêuticos e aeróbicos também contribuem significativamente para a prevenção e controle da hipertensão, podendo ser também uma das estratégias utilizadas para reabilitação de sequelas instaladas do AVC ${ }^{11}$.

Tem sido documentada por meio de estudos epidemiológicos uma associação entre o baixo nível de atividade física ou condicionamento físico com a presença de HAS. E, por outro lado, grandes ensaios clínicos aleatorizados e metanálises não deixam dúvidas quanto ao efeito benéfico do exercício sobre a pressão arterial de indivíduos hipertensos leves e moderados. Isto é, o treinamento físico reduz significativamente a pressão arterial em pacientes com HAS ${ }^{12}$. 
A prática regular de exercício físico conduz a importantes adaptações cardiovasculares, reduzindo a pressão arterial sanguínea em indivíduos hipertensos. Felizmente, sabe-se que $75 \%$ dos pacientes hipertensos, que realizam exercício físico regular, diminuem os níveis de PA, o que faz com que essa conduta seja considerada importante ferramenta no tratamento da HAS ${ }^{13}$.

Em diversas situações durante a vivência no grupo, direcionou-se pela ferramenta da clínica ampliada, que tem como objetivo ajustar os recortes teóricos de cada profissional às necessidades dos usuários.

A clínica ampliada consiste basicamente na articulação e diálogo de diferentes saberes para compreensão dos processos de saúde e adoecimento e na necessidade de inclusão dos usuários como cidadãos participantes das condutas em saúde, inclusive da elaboração de seu projeto terapêutico ${ }^{14}$.

A construção da clínica ampliada é justamente a transformação da atenção individual e coletiva, de forma a possibilitar que outros aspectos do sujeito, que não apenas o biológico possam ser compreendidos e trabalhados ${ }^{15}$.

Muitos dos pacientes além de hipertensos chegavam até o grupo com problemas de saúde diferenciados, como algias crônicas. Essas situações não foram negligenciadas, embora o objetivo fosse a prevenção e o controle da HAS. No grupo haviam usuários apresentando queixas dolorosas, portanto o trabalho não poderia se resumir em educação em saúde e praticas corporais generalizadas. Sendo assim, foi preciso adequar as estratégias dos profissionais ao perfil dos usuários, por isso o trabalho foi realizado em parceria para assim desenvolver suas potencialidades, tentando sempre visualizar os pacientes de forma integral e humanizada.

Estes usuários receberam atenção diferenciada, devido à limitação e à contraindicação de alguns movimentos. Foi praticado dentro do grupo exercícios que contribuíssem para o alívio destes processos álgicos. Concomitantemente, estes pacientes foram inseridos nas redes de assistência à saúde do SUS para aguardar o tratamento fisioterapêutico.

O grupo tinha, também, outro perfil de usuários: os não hipertensos, que desejavam prevenir a HAS. Com estes a educadora física realizava uma série de exercícios, visando prevenir a patologia. Em outros casos a hipertensão estava relacionada ao fator emocional daquele paciente, então se acionava o serviço de psicologia da equipe, que a partir desta solicitação responsabilizase por este usuário. O primeiro aspecto facilitador foi à interação entre a equipe de NASF e coordenação da Unidade Básica de Saúde, que acolheu prontamente a missão da equipe de prevenir doenças e promover saúde da população.

O serviço de Odontologia da equipe 1 e 2 contribuiu em dois encontros com palestras educativas. Os outros profissionais da saúde não aderiram à proposta de participarem do grupo, provavelmente por falta de sensibilização em trabalhar com as propostas de prevenção e promoção da saúde ou por a equipe do NASF não ter conseguido repassar a importância das atividades desenvolvidas.

Nesse contexto, a exceção foram os ACS, que independente da equipe a que pertenciam integraram-se ao grupo, comparecendo com frequência e incentivando seus respectivos usuários a participarem assiduamente do grupo.

O comprometimento dos usuários com a equipe de saúde tornou-se um estímulo importante para a sequencia das atividades propostas. $O$ interesse na realização das práticas corporais, a troca de saberes proporcionada pela educação em saúde e educação popular facilitavam o desempenho das competências das ações do NASF.

Uma das principais dificuldades enfrentadas para desempenho das atividades de competência da fisioterapia no NASF foi a falta de conhecimento de profissionais da saúde e usuários em relação ao papel do fisioterapeuta na Atenção Básica. Muitos profissionais acreditavam que as atividades eram desnecessárias e que o trabalho do fisioterapeuta deveria ser priorizado nos atendimentos de pacientes acamados com disfunções respiratórias, traumatológicas, neurológicas e dentre outras, ou seja, o exercício de conduta puramente curativa e reabilitadora.

Outra dificuldades era assistir e dar continuidade ao atendimento de pacientes cadeirantes ou que apresentavam alguma limitação fisica. Estes pacientes eram os menos assíduos, pois normalmente encontravam dificuldades de acesso devido à má pavimentação das ruas e calçadas ou à necessidade de utilizar o transporte público, culminando no não comparecimento em muitos encontros.

Acredita-se ser possível desenvolver um trabalho de qualidade na Atenção Básica, mesmo com tantas dificuldades, se forem utilizadas as ferramentas corretas e houver uma interação com os gestores e as equipes de Estratégia de Saúde da Família, tendo como consequência resultados positivos nos indicadores de saúde.

\section{Considerações Finais}

A experiência de promoção de saúde no Grupo Revivendo proporcionou a ampliação dos conhecimentos e de estratégias de promoção e prevenção da saúde, para todos os envolvidos, pois mostrou a relevância da utilização da criatividade e da renovação por parte das equipes de NASF de da ESF no planejamento de uma assistência de saúde integral aos participantes. 
Considera-se as ações do Grupo Revivendo exitosas no trabalho de intervenção e prevenção realizado pelas equipes na atenção básica e da percepção das pessoas em relação ao seu estado de saúde. Houve melhora nos resultados de controle da PAS, do comprometimento com o tratamento farmacoterapêutico e maior interesse na realização nas práticas terapêuticas corporais. Sugere-se que o trabalho do grupo seja contínuo, com ampliação para outras área de cobertura da unidade de saúde com a participação de um maior número de profissionais voltados para as atividades de prevenção das doenças.

\section{Referências Bibliográficas}

1. Brasil. Ministério da Saúde. Portaria GM/ MS 2.488, de 21 de outubro de 2011. Aprova a Política Nacional de Atenção Básica, estabelecendo a revisão de diretrizes e normas para a organização da Atenção Básica, para a Estratégia Saúde da Família (ESF) e o Programa de Agentes Comunitários de Saúde (PACS). Diário Oficial da União. Brasília, 2011.

2. Fortaleza, Secretaria Municipal de Saúde. Relatório de Gestão 2012 ,Fortaleza, 2013.

3. Araújo KPS, Toniolli TR, Drummond AA. A fisioterapia em pacientes hipertensos da Estratégia Saúde da Família no Recanto das Emas - Distrito Federal. Revista Eletrônica Gestão \& Saúde. 2012; 3(2): 773-87.

4. Barbosa EG, Ferreira DLS, Furbino SAR. Experiência da fisioterapia no Núcleo de Apoio à Saúde da Família em Governador Valadares, MG. Fisioterapia em Movimento. 2010; 23 (2): 323-30.

5. Mângia, EF \& Lancman, S. Núcleos de Apoio à Saúde da Família: integralidade e trabalho em equipe multiprofissional. Revista de Terapia Ocupacional da Universidade de São Paulo. 2008 19(2), ii.

6. Moura,AA, Nogueira, MS. Enfermagem e educação em saúde de hipertensos: revisão da literatura. J Manag Prim Health Care 2013; 4(1):36-41.

7. Brasil. Secretaria de Atenção à Saúde, Diretoria da Atenção Básica, Ministério da Saúde. Cadernos de Atenção Básica: hipertensão arterial sistêmica para o SUS. Brasília (Brasil): Ministério da Saúde.2006.

8. Lima MMO, Britto RR, Baião EA, Alves GS, Abreu CDG, Parreira VF. Exercício aeróbico no controle da hipertensão arterial na pós-menopausa. Fisioterapia em Movimento. 2011; 24(1): 23-3.

9. Novais BKLO, Brito GEG. Percepções sobre o Trabalho do Fisioterapeuta naAtenção Primária. Revista deAPS. 2011; 14(4): 424-34. 10. Gomes AMA, Sampaio JJC, Carvalho MGB, Nations MK, Alves MSCF. Código dos direitos e deveres da pessoa hospitalizada no SUS: o cotidiano hospitalar na roda de conversa. Interface. 2008; 12(27): 773-82

11. Ferreira AP, Ferreira CB, Campos BRM, Samy GCP, Morais PP. Efeito de diferentes intensidades de exercício aeróbio na resposta pressórica de 24 horas em mulheres normotensas. Journal of the Health Sciences Institute. 2011; 29 (1): $62-6$.

12. Baldissera VDA, Carvalho MDB, Pelloso SM. Adesão ao Tratamento não-farmacológico entre hipertensos de um centro de saúde escola. Revista Gaúcha de Enfermagem. 2009; 30 (1): 27-32.

13. Oliveira, K PC., Vieira, EDL., Oliveira, JD, Oliveira, KD, Lopes, FJG, Azevedo, LF. Exercício aeróbio no tratamento da hipertensão arterial e qualidade de vida de pacientes hipertensos do Programa de Saúde da Família de Ipatinga. Rev Bras Hipertens. 2010 17(2), 78-86.

14. Brasil. Ministério da Saúde. Secretaria de Atenção à Saúde. Núcleo Técnico da Política Nacional de Humanização. Clínica ampliada, equipe de referência e projeto terapêutico singular / Ministério da Saúde, Secretaria de Atenção à Saúde, Núcleo Técnico da Política Nacional de Humanização - 2. ed. - Brasília: Ministério da Saúde, 2007.

15. Cunha, GT. A Construção da Clínica Ampliada na Atenção Básica São Paulo: Hucitec, 2005. 


\section{Marcielly dos Santos Maciel}

Endereço para correspondência - Rua: Alexandre Barauna, $n^{\circ}$ 1330, Bairro: Rodolfo Teófilo, CEP - 60430-160, Cidade: Fortaleza, CE, Brasil.

E-mail: marcisantos@bol.com.br

Lattes: http://lattes.cnpq.br/0426435419509945

Márcia Oliveira Coelho Campos - lidiavaladas@hotmail.com

Lidia Audrey Rocha Valadas Marques - lidiavaladas@gmail.com

Edilson Martins Rodrigues Neto - edilsonmrneto@gmail.com

Mara Assef Leitão Lotif - mara_lotif@hotmail.com

Emerson Dias Ponte - emersondiasp@gmail.com

Enviado em 25 de março de 2014.

Aceito em 31 de outubro de 2014. 